\title{
A Framework for Teaching Conceptual Modeling and Metamodeling Based on Bloom's Revised Taxonomy of Educational Objectives
}

\author{
Dominik Bork \\ University of Vienna, Research Group Knowledge Engineering \\ dominik.bork@univie.ac.at
}

\begin{abstract}
Conceptual modeling and metamodeling are vital parts in computer and information science study programs at tertiary institutions. Currently, teachers are struggling in ensuring that their teaching approach is comprehensive and in identifying application domains that motivate students, and show that the value of models exceeds pure representative means. This paper uses Bloom's revised taxonomy of educational objectives as a foundation to define a framework for comprehensive teaching of conceptual modeling and metamodeling. The introduced framework is used to evaluate the comprehensiveness of a Smart City teaching case which has been taught at the Next-generation Enterprise: Modeling in the Digital Age Summer School. The contribution of this paper is threefold: First, a generic framework for comprehensive teaching of conceptual modeling and metamodeling is proposed; Second, a Smart City teaching case is reported; Third, the evaluation of the teaching case leads to a discussion on how to improve teaching of conceptual modeling and metamodeling in the future.
\end{abstract}

\section{Introduction}

Education in conceptual modeling and metamodeling is of increasing interest currently $[1,2,3,4]$. Its relevance is not at last underpinned by the invitation of the 'Software Engineering Education and Training' track including the Teaching Conceptual Modeling (TeCoMo) ${ }^{1}$ mini-track at the Hawaii International Conference on System Sciences and the Teaching \& Learning Conceptual Modeling (TLCM) workshop at the International Conference on Practicing Enterprise Modeling ${ }^{2}$.

"Conceptual modeling research has a long tradition

\footnotetext{
${ }^{1} \mathrm{TeCoMo}$ homwpage [online], https://tecomo2019. wordpress . com/ last checked: 19.09.2018

${ }^{2}$ TLCM workshop [online], http://poem2018.omilab. org/workshops\# last checked: 19.09 .2018
}

in the scientific discipline of Business and Information Systems Engineering." [5] Methods such as Business Process Modeling and Notation (BPMN) and Unified Modeling Language (UML) are vital cornerstones in the education of computer science and information science students at tertiary institutions [6]. On the one side, modeling capabilities are increasingly required in industry, on the other, the underlying capability of abstraction is a foundational capability that helps students to abstract from problem/situation-specific instances toward the design of solutions that can be applied to a class of problems. In the context of metamodeling, abstraction refers to the reduction of domain complexity for a specific purpose. The result of metamodeling is a conceptual modeling language (i.e., a metamodel) that is capable of being used in numerous instances for specific purposes.

Mylopoulos defines conceptual modeling, as "the activity of formally describing some aspects of the physical and social world around us for purposes of understanding and communication" [7]. In order for students to apply conceptual modeling and train their proficiency, two prerequisites need to be fulfilled: a) conceptual modeling needs to be taught and testes comprehensively; and b) appropriate application cases need to be given [8] in order to keep students motivated. Smart Cities are an emerging field in research and development. When putting yourself into the position of a city manager, assigned the task to transform a city into a Smart City, a plethora of possibilities but also challenges come to play. This complexity combined with the design possibilities constitute a Smart City an interesting learning environment for conceptual modeling and metamodeling.

This paper investigates the educational and pedagogical foundations of teaching conceptual modeling and metamodeling. Therefore, it first introduces the taxonomy of educational objectives as proposed by [10] as a revision of the initial dimensions by Bloom et al. [11]. These foundations will then be used to establish a generic framework for the 


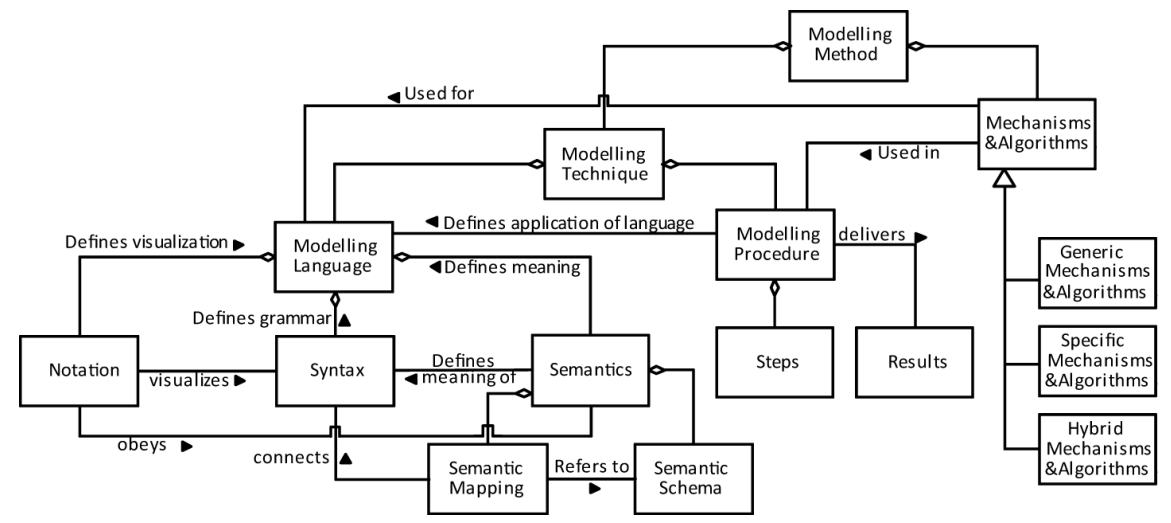

Figure 1. Components of modeling methods [9]

comprehensive teaching of conceptual modeling and metamodeling. This framework is then used to evaluate to what extent the Smart City case provides an environment to teach conceptual modeling and metamodeling along all of Bloom's dimensions. We report on our experience of teaching the Smart City case with Master and $\mathrm{PhD}$ students at the Next-generation Enterprise: Modeling in the Digital Age Summer School Series $(N E M O)^{3}$. The Smart City teaching case is composed of multiple scenarios, addressing the innovation challenges stressed in [12]: Smart City Planning, Smart City Management, and Smart City Operation. From this, we map the individual student tasks within the case to the proposed educational framework. From this mapping, conclusions and future research directions are drawn that have the potential to guide the creation of new teaching cases.

The research question guiding this paper is: "How to teach conceptual modeling and metamodeling comprehensively, i.e., including all dimensions of Bloom's revised taxonomy?". This paper's contribution is of primary interest for researchers, interested in educational and generic aspects of conceptual modeling and metamodeling as well as for lecturers, interested in evaluating their current courses and possibly improving them in the future.

The rest of the paper is organized as follows. The theoretical foundations are introduced in Section 2. Section 3 introduces the revised version of Bloom's taxonomy on educational objectives. In Section 4, Bloom's taxonomy is related to the generic aspects of educating conceptual modeling and metamodeling, thereby defining a comprehensive educational framework. This framework is used in Section 5 to evaluate the comprehensiveness of the Smart City teaching case. Section 6 concludes this paper and points to some future research directions.

\footnotetext{
${ }^{3}$ NEMO Summer School [online]: http://nemo.omilab.org, last visited: 19.09 .2018
}

\section{Foundations}

The relevant foundations this research builds upon are introduced in the following: domain-specific conceptual modeling (Section 2.1), Open Models Laboratory (Section 2.2), and the NEMO Summer School Series (Section 2.3).

\subsection{Domain-specific Conceptual Modeling}

Existing systems and human beings are limited when it comes to planning, management, and operation of complex systems like Smart Cities (cf. [13]). Conceptual modeling approaches can assist, support, or even substitute human activities. These approaches not only cope with the increasing complexity by providing structuring, planning, and analysis capabilities. Moreover, given that they are based on a formal specification [14], they establish a knowledge base that can be processed by machines.

The creation of valid models is guided by a modeling method. As defined by [9], three building blocks constitute a modeling method (see Figure 1): The modeling language, defining the syntax, i.e., the elements and the rules constraining allowed combinations thereof; the semantics, i.e., the meaning of syntactic elements; and the notation, i.e., the graphical visualization of syntactic elements. The way modelers are intended to utilize a given modeling language is specified in the modeling procedure. Model processing functionality is specified in Mechanisms \& Algorithms, e.g., simulation or transformation of models.

General purpose modeling languages like BPMN and UML are widely used in industry for predefined application scenarios. With emerging and upcoming domains like Smart Cities, however, such standards are not adequate for all domain-specific requirements and their necessary depth of conceptual specialization. Hence, components of modeling methods need to be 
agilely adopted to the specific requirements of such domains. Modeling methods in this regard "represent a clearly more productive instrument for describing and analyzing problems as well as for designing systems" [15, p. 155]. An overview of domain-specific conceptual modeling methods is provided by [16].

\subsection{The Open Models Laboratory (OMiLAB)}

The Open Models Laboratory (OMiLAB) [17] is a virtual and physical environment for modeling method engineering and modeling tool development following the Agile Modeling Method Engineering (AMME) approach [18]. The OMiLAB is an international community [19] of modeling enthusiasts interested in developing novel modeling methods by using the open metamodeling platform ADOxx ${ }^{4}$. ADOxx is the technological enabler for the OMiLAB. It is an open use platform that is heavily used in academia and industry for the development of modeling tools $[20,16]$.

\subsection{The NEMO Summer School Series}

Dissemination and validation of OMiLAB methods and tools can take place as part of the NEMO Summer School Series. NEMO was initiated in 2014, aiming to provide an educational forum for Master and $\mathrm{PhD}$ students interested in conceptual modeling. Each year 50 to 70 international students participate in lectures and exercises focusing on foundations, applications and technologies of conceptual modeling.

At NEMO, theoretical lectures are complemented by exercises, enabling the students to transform their theoretical knowledge towards practical application in challenging and interesting domains. Since 2015, one exercise block comprising multiple sessions addresses Smart City challenges by means of conceptual modeling and metamodeling [21, 22]. The relevant parts of this Smart City case will be briefly introduced in Section 5 .

\section{Bloom's Taxonomy of Educational Objectives}

In 1949, a group of measurement specialists established a coherent set of principles aiming to foster the creation of comprehensive and comparable examinations. As a result, the initial Bloom taxonomy of learning objectives [11] comprising the categories: Knowledge, Comprehension, Application, Analysis, Synthesis, and Evaluation was published. A second group of researchers then proposed a revised version of the taxonomy [10], aiming to address some

\footnotetext{
${ }^{4} \mathrm{ADOxx}$ metamodeling platform [online] http://www.adoxx.org, last visited: 20.09 .2018
}

identified shortcomings of the initial version, which will be used throughout this paper. [10] introduced a two-dimensional schema for the assessment of learning objectives: the knowledge dimension and the cognitive process dimension. Both will be further detailed in the following.

For the knowledge dimension, learning objectives can be distinguished based on their subject matter. In total, four categories can be distinguished: [10, p. 214]:

Factual Knowledge The basic elements that students must know to be acquainted with a discipline or solve problems in it. This category contains the sub-categories knowledge of terminology and knowledge of specific details and elements.

Conceptual Knowledge Interrelationships between the basic elements within a larger structure that enable them to function together. This category contains the sub-categories knowledge of classification and categories, knowledge of principles and generalization, and knowledge of theories, models, and structures.

Procedural Knowledge How to do something; methods of inquiry; and criteria for using skills, algorithms, techniques, and methods. This category contains the sub-categories knowledge of subject-specific skills and algorithms, knowledge of subject-specific techniques and methods, and knowledge of criteria for determining when to use appropriate procedures.

Metacognitive Knowledge Knowledge of cognition in general as well as awareness and knowledge of one's own cognition. This category contains the sub-categories strategic knowledge, knowledge about cognitive tasks, including appropriate contextual and conditional knowledge, and self-knowledge.

In the cognitive process dimension, six categories can be distinguished. These categories specify what is to be done with or to a specific content to be learned. All six categories establish a cumulative hierarchy ranging from basic recall to higher educational objectives like evaluate and create [23]. Consequently, mastering a certain cognitive process category implies mastering the previous one as a prerequisite [10, p. 215]:

Remember Retrieving relevant knowledge from long-term memory. This category contains the sub-categories Recognizing and Recalling.

Understand Determining the meaning of instructional messages, including oral, written, and graphic 
communication. This category contains the sub-categories Interpreting, Exemplifying, Classifying, Summarizing, Inferring, Comparing, and Explaining.

Apply Carrying out or using a procedure in a given situation. This category contains the sub-categories Executing and Implementing.

Analyze Breaking material into its constituting parts and detecting how the parts relate to one another and to an overall structure or purpose. This category contains the sub-categories Differentiating, Organizing, and Attributing.

Evaluate Making judgments based on criteria and standards. This category contains the sub-categories Checking and Critiquing.

Create Putting elements together to form a novel, coherent whole or make an original product. This category contains the sub-categories Generating, Planning, and Producing.

\section{Relating Conceptual Modeling and Metamodeling to Bloom's Taxonomy}

This Section relates the dimensions and categories of the revised Bloom Taxonomy as introduced previously to conceptual modeling (Section 4.1) and metamodeling (Section 4.2). The goal is to establish generic comprehensive educational frameworks for those fields.

\subsection{Teaching Conceptual Modeling Comprehensively}

In the following, a mapping between the knowledge dimensions and conceptual modeling is established.

Factual Knowledge This category refers to the factual knowledge of conceptual modeling, e.g., knowledge about the constituents of conceptual models, their purposes, and goals. In the context of one modeling language, factual knowledge comprises the knowledge about the elements that constitute the modeling language with their semantics and notation.

Conceptual Knowledge This category refers in a narrow scope to the valid combinations of language elements and the structuring of large models, e.g., using submodels. On a broader scope, this category refers to the capability of comparing different modeling languages based on their fitness to a given (set of) purpose(s).
Procedural Knowledge This category considers the modeling procedure of a modeling method, i.e., the sequence of steps necessary to create valid models while applying a modeling language. This category refers to the ability of a learner to decide which modeling method to apply in a given scenario. Thus, conceptual knowledge of different modeling methods is combined with the knowledge on criteria for selecting the most appropriate method in a given context.

Metacognitive Knowledge This category incorporates strategic thinking. Thus, learners enrich the procedural knowledge with aspects, such as who will be the addressee of the model, what is she/he doing with the model, how much time and resources are available to create the model, how experienced am I in a given modeling method.

The described mapping of the knowledge dimensions shows, that when considering the broader notion of a modeling method (see Figure 1), different parts of modeling methods are dominant for different knowledge dimensions. This particularly holds for the procedural knowledge dimension and the modeling procedure.

Similar observations can be drawn when looking at the relation between conceptual modeling teaching and the cognitive process dimensions. Table 1 summarizes the mappings. Besides the mapping, the table also discusses relevant exercises applicable for testing the conceptual modeling knowledge of a learner.

\subsection{Teaching Metamodeling Comprehensively}

With the uprise of domain-specific modeling methods [15, 16], metamodeling - i.e., the process of designing a new modeling language - becomes also a part of teaching conceptual modeling, especially in graduate courses. Metamodels are therefore the artifact of interest in the following. Consequently, learning objectives are specified by mapping the revised Bloom taxonomy dimensions to metamodeling. In the following, first the knowledge dimensions for metamodeling are described.

Factual Knowledge Foundational aspects of metamodeling, e.g., the constituents of a metamodel like object types, relationship types, model types, and attributes (cf. [28]). Foundations regarding notational aspects, e.g., Physics of Notation [36], regarding semantics [14], and regarding the techniques to specify metamodels [37] are also considered here. 
Table 1. Cognitive Process Dimension Mapped to Conceptual Modeling

\begin{tabular}{|c|c|}
\hline Dimension & onceptual Modeling \\
\hline Remember & $\begin{array}{l}\text { The goal is to have knowledge of the syntactical, semantical, and notational aspects of a language, } \\
\text { as well as the modeling procedure and mechanisms \& algorithms of a modeling method. }\end{array}$ \\
\hline Understand & $\begin{array}{l}\text { In order to understand a conceptual model, learners need to know the meaning of the single elements } \\
\text { of a model as well as the meaning of the relationships between those elements. Consequently, this } \\
\text { level concentrates on the semantical aspects of a modeling language. }\end{array}$ \\
\hline Apply & $\begin{array}{l}\text { Learners need to apply a modeling method in a way that serves a certain purpose or solves a problem } \\
\text { at hand. This level concentrates on the whole modeling method. Learners might decide among } \\
\text { several visualized models, which one fits to a certain case scenario, or they might use a predefined } \\
\text { model to generate code (e.g., generate SQL code from ER models), or transform it into a different } \\
\text { model following a predefined procedure by applying a set of model transformations. }\end{array}$ \\
\hline Analyze & $\begin{array}{l}\text { The analysis of a conceptual model can be on syntactic and/or semantic level, i.e., analyzing the } \\
\text { usage of certain syntactic elements, or the semantic analysis of larger models, optionally decomposed } \\
\text { into interlinked submodels. Consequently, this level concentrates on syntax and semantics of a } \\
\text { modeling method. }\end{array}$ \\
\hline Evaluate & $\begin{array}{l}\text { The evaluation level analyzes e.g., also the syntax and semantics of a model, but with the objective } \\
\text { of relating the analysis results to given criteria. Syntactic evaluations on conceptual models can } \\
\text { be performed e.g. by using cardinalities and the allowed relationships between classes. Semantic } \\
\text { evaluations can analyze, whether a given model correctly and comprehensively represents some } \\
\text { aspects of the real world or a given case study, or whether two or more models are consistent [24, 25]. } \\
\text { An extensive evaluation of model quality can be referred to the quality criteria of [26]. }\end{array}$ \\
\hline Create & $\begin{array}{l}\text { The creation of a new conceptual model. Consequently, the focus of this level is on all aspects of a } \\
\text { modeling method. Learners need to be able to choose the correct syntactic elements that fit to the } \\
\text { semantics of the system under study, model them with their correct notation, and relate them to other } \\
\text { elements using the correct relation classes. For more complex modeling methods, the creation might } \\
\text { also involve following a predefined procedure and applying mechanisms \& algorithms. }\end{array}$ \\
\hline
\end{tabular}

Conceptual Knowledge This category refers to how metamodels are organized in meta-hierarchies [27] and how concepts such as generalization and inheritance can be employed in metamodel design. Moreover, knowledge on how to realize metamodels on a metamodeling platform are relevant [38, 20].

Procedural Knowledge Procedures that support the development of metamodels. Approaches dealing with the composition of existing metamodels [39], the development of domain-specific modeling languages [15], metamodel metrics [29] as well as agile [40] and model-driven [41] approaches for modeling method engineering are relevant.

Metacognitive Knowledge Learners are able to choose a specific metamodeling technique based on context, purpose (e.g., for tool development, documentation, or model transformation), and available resources. Experience from previous metamodeling tasks are also reflected and integrated into the current task.
Table 2 summarizes the mapping between metamodeling and the cognitive process dimension of Bloom's revised taxonomy. Besides describing the mapping, some means of testing the relevant knowledge on learner side are given.

\section{Evaluating the Smart City Case}

A teacher in conceptual modeling should always aim to incorporate realistic, challenging, and motivating application cases. Consequently, we created a new teaching case that centers a Smart City and explores different possibilities of utilizing domain-specific conceptual modeling in such a domain. This Section briefly motivates the choice of a Smart City in Section 5.1. Afterwards, the structure and content of the different Smart City scenarios are described in Section 5.2. Section 5.3 then evaluates the educational objectives of these scenarios against the teaching frameworks introduced in the previous Section. Eventually, Section 5.4 discusses the results and provides ideas for improving the Smart City case. 
Table 2. Cognitive Process Dimension Mapped to Metamodeling

\begin{tabular}{l|l} 
Dimension & Metamodeling \\
\hline Remember & $\begin{array}{l}\text { This category tests, whether learners have the basic knowledge of the foundations of metamodeling. } \\
\text { This includes knowledge about meta-metamodels, metamodel hierarchies [27], and the constituents } \\
\text { of metamodels [28]. }\end{array}$ \\
\hline Understand & $\begin{array}{l}\text { This level requires understanding the consequences of metamodel design decisions for the } \\
\text { expressiveness of the resulting modeling language [29]. Learners should moreover understand the } \\
\text { domain for which a certain metamodel is designed for. }\end{array}$ \\
\hline Apply & $\begin{array}{l}\text { Learners are able to apply metamodels in different scenarios like model weaving [30], metamodel } \\
\text { interoperability [31], or consistency management [32, 33]. }\end{array}$ \\
\hline Enalyze & $\begin{array}{l}\text { In this level, learners shall be able to analyze metamodels, e.g. by applying a set of metrics [29, 34]. } \\
\text { Learners shall be able to evaluate a metamodel, e.g. targeting at assessing the metamodel quality by } \\
\text { means of empirical studies [35]. Thus, learners shall be able to evaluate whether a given metamodel } \\
\text { fosters understandability or whether it is correct, concise, and consistent [35]. }\end{array}$ \\
\hline Create & $\begin{array}{l}\text { Learners shall be able to create new metamodels, e.g., for a specific domain. Thus, learners might } \\
\text { be challenged with a domain description and/or some requirements in natural language and should } \\
\text { create a metamodel as a possible solution. }\end{array}$ \\
\hline
\end{tabular}

\subsection{Motivation for a Smart City Case}

Smart Cities are continuously evolving and with every new technological improvement, possibilities and challenges for/in a Smart City emerge. It is therefore not surprising, that a precise specification of a Smart City is still lacking [42] but cases, also driven by the industry are emerging [43, 44]. Planning, management, and operation of Smart Cities requires innovation and abstraction to cope with the complexity and design novel solutions to upcoming needs. Conceptual modeling can play a vital role in this regard (cf. [12, p. 36]).

The Smart City case in this paper contributes to the research framework proposed by [42, 45] in the fields of technology, people and communities and natural environment. Following the vision of [46], this paper contributes to the intelligent transport systems, smart mobility, logistics and technology, quality and sustainability of living, and ecosystem: sustainable environment, renewable energy and other resources. Considering the research challenges by [47], this paper proposes a teaching case and an evaluation towards: i) "how governmental agencies may use the IoT to serve citizens in the smart city of tomorrow" [47, p. 1538].

\subsection{Structure and Content of the Case}

We considered the situation of a planner, responsible for planning, management and operating a Smart City. Accordingly, we specified multiple scenarios, focusing on tasks of the Smart City planner. The underlying goals were: i) to familiarize students with the domain; ii) to guide them through an exercise of "abstracting" this domain, where they need to isolate relevant concepts for a given scenario; iii) to guide them through an exercise of formally and graphically representing this abstraction by designing a domain-specific modeling language; iv) to make them aware of different means of how the models may be processed (queries, simulation); and v) to guide them through implementing all this using a metamodeling platform.

The Smart City scenarios cover aspects from pure application of an existing conceptual modeling language up to the creation of new metamodel concepts by extending a given minimalistic Smart City metamodel. The scenarios are taught in three sessions. The content of the scenarios evolved in several years of teaching at the NEMO Summer School Series. In the following, we will briefly introduce the content and learning tasks of each session. More details on the content of the scenarios can be found in [21,22].

Session 1 - Planning a Smart City The first session briefly introduced the Smart City as an application domain and conceptual modeling as well as metamodeling as approaches to cope with complexity. Afterwards, the learners were asked to do the following exercises: S1E1-Create a new modeling class, learners were asked to abstract from a Smart City and to define a new concept as an extension of the provided minimalistic metamodel; S1E2-Realize a static graphical visualization, learners were asked to define and implement a graphical visualization for their new modeling class; S1E3-Realize a dynamic graphical visualization, learners were asked to define 
and implement an attribute-dependent visualization of their new modeling class; S1E4-Create a new Relation Class, learners were asked to define a new relation class, connecting two (or more) existing metamodel concepts; S1E5-Create Smart City models, eventually, this session closed with the task to create Smart City models by using the new modeling and relation classes.

Session 2 - Analyzing a Smart City The second session briefly introduced how conceptual Smart City models might be used in order to analyze and monitor Smart Cities. The learners were given a set of complex Smart City models and four tasks. Each task was separated into three blocks: First, a theoretical introduction to the relevant query theory was given. Second, a demonstration of an application of such a query was performed. Third, the learners were asked to do some hands-on work.

The following tasks were part of the second session: S2E1-Standardized Queries, the learners were introduced to the basic standardized ADOxx queries and asked to apply them on the provided models; S2E2-Combination of Standardized Queries, learners were introduced to the logical operators for combining simple queries toward more complex model analysis and asked to apply them to the given models; S2E3-User-defined Queries, the learners were asked to learn parts the ADOxx Query Language (AQL) and to use it in order to create new queries that should be applied to the provided models; S2E4-Configurable Queries, learners were asked to follow a predefined procedure in order to create new configurable queries for the Smart City models.

Session 3 - Simulating a Smart City The third exercise session concentrated on simulating the Smart City models. ADOxx provides three kinds of simulations: Path Analysis, searching and quantifying different paths throughout a graph-based model; Capacity Analysis, quantifying the required personnel and machinery to execute a set of process models; and Workload Analysis, simulating the workload of the given personnel and machinery when executing a set of process models. For each task within this exercise, learners were first introduced to the necessary simulation theory before they were asked to use this knowledge while solving practical tasks.

The third exercise session comprised: S3E1-Perform a Path Analysis, learners were asked to execute a path analysis on a provided Smart City model and to interpret the results; S3E2-Model Preparation for Capacity/Workload Analysis, this task required that the learners link personnel and machinery as performers to process model activities; S3E3-Perform Capacity Analysis, learners were asked to configure and execute a capacity analysis on the prepared models; S3E4-Perform Workload Analysis, learners were asked to configure and execute a workload analysis on the prepared models.

\subsection{Evaluating the Comprehensiveness}

In the following, we will use the frameworks for comprehensive teaching of conceptual modeling (see Tables 1) and metamodeling (see Table 2) to evaluate the comprehensiveness of the Smart City case. Table 3 summarizes the results of the evaluation. For each exercise, we mapped the tasks to the corresponding highest cognitive process dimension. Noteworthy, as stated in Section 3, a task assigned to the analyze dimension also includes remember, understand, and apply. For example, task S2E3 and S2E4 - the creation of queries - obviously also involves: remembering the foundations of the modeling language, understanding the query theory, and applying the query to a model.

The tasks of the first exercise session concentrate on the create category of the cognitive process dimension. All five tasks involve the creation of extensions to an existing metamodel. This requires factual knowledge on the foundations of metamodeling as well as conceptual knowledge for the domain abstraction and the interplay of the different metamodel elements.

The tasks of the second exercise session are distributed among several cognitive process and knowledge dimensions. Four tasks relate to the analyze and the create category whereas two relate to the apply category. Thus, learners not only created new queries as a part of metamodeling education, they moreover applied these queries in order to analyze Smart City models as a part of conceptual modeling education. In this exercise, also procedural knowledge was required for the first time as the creation of queries in ADOxx needs to follow a predefined procedure.

The third exercise session focused on the cognitive process categories apply (three tasks) and analyze (three tasks). Application of the simulation functionality required procedural knowledge as a predefined sequence of steps needs to be followed. The analysis then required factual knowledge in case of the path analysis, and conceptual knowledge for the capacity and workload analysis. The reason for this differentiation is that path analysis solely relies on one model, whereas the latter two simulations require the linkage of several models - in the Smart City case graph-based models describing procedural aspects needed to be linked to static models describing the available machinery and personnel responsible for executing the procedure. Consequently, the creation of the necessary models in this task required also conceptual knowledge. 
Table 3. Evaluation Results

\begin{tabular}{c|c|c|c|c|c|c}
\multirow{2}{*}{$\begin{array}{c}\text { Knowledge } \\
\text { Dimension }\end{array}$} & \multirow{2}{*}{ Remember } & Understand & Apply & Analyze & Evaluate & Create \\
\hline $\begin{array}{c}\text { Factual } \\
\text { Knowledge }\end{array}$ & & & S2E1 & S2E1, S2E2, & & S2E3, S3E1 \\
\hline $\begin{array}{c}\text { Conceptual } \\
\text { Knowledge }\end{array}$ & & & S2E2 & S2E4, S3E3, & & S1E3, S1E4, \\
\hline $\begin{array}{c}\text { Procedural } \\
\text { Knowledge }\end{array}$ & & & S2E2, S3E1, & & S1E5, S2E3, S2E4 \\
\hline $\begin{array}{c}\text { Metacognitive } \\
\text { Knowledge }\end{array}$ & & & S3E3, S3E4 & & & S1E3, S1E4, \\
\hline
\end{tabular}

\subsection{Discussion}

The previous evaluation revealed, that the different exercises of the Smart City case have quite different foci and educate different aspects of the teaching frameworks. Interestingly, the majority of tasks concentrate on the creation category, i.e., the creation of a conceptual model, the creation of a model query, or the creation of a metamodel extension.

Mere consideration of the categories remember and understand are not part of the Smart City tasks, but subsumed in more compex tasks. Metacognitive knowledge is not considered because of the context within which the Smart City case is being taught. NEMO comprises participants from divers backgrounds (i.e., computer and information science) with heterogeneous knowledge and expertise. In contrast to regular classes in undergraduate or graduate level at universities, NEMO has neither the possibility nor the objective to educate and test the metacognitive knowledge which would require more than the allocated three exercises sessions.

Based on the evaluation, we believe it will be interesting to think about new scenarios in a Smart City that incorporate the evaluation category and the metacognitive dimension. If more time can be allocated to the Smart City case, learners could compare and evaluate different metamodels for a Smart City. Discussing strengths and weaknesses of two metamodels will surely be of benefit for educating the next generation of metamodelers. Moreover, aspects such as model quality are completely neglected as of now. The evaluation of different Smart City models according to quality metrics will foster the education of modeling experts.
During this research, we realized, that metamodelling tasks coincide with tasks on the conceptual modeling level because of their conceptual relatedness. For example, when creating a metamodel - by definition - we also create a model (of a modeling language). From the conceptual modeling perspective, one could argue that while creating models, an application, an analysis, or even an evaluation of the corresponding metamodel is performed. These are aspects that deserve further consideration in our future research. The two frameworks introduced in Section 4 however aim for generality and shall enable independent adoption. They establish a first step toward the design of comprehensive conceptual modeling and metamodeling courses.

\section{Conclusion}

The paper at hand aimed to answer the research question "How to teach conceptual modeling and metamodeling comprehensively?" In order to answer this question, this paper introduced the relevant foundations including Bloom's revised taxonomy on learning objectives. Based on this taxonomy, two generic educational frameworks for comprehensive teaching of conceptual modeling and metamodeling have been proposed.

The frameworks have later on been used to evaluate the comprehensiveness of multiple scenarios of a Smart City teaching case taught at the Next-generation Enterprise: Modeling in the Digital Age Summer School. The evaluation revealed, that not all knowledge dimension categories are covered in the case. It moreover pointed to interesting aspects to consider in future extensions of the case, i.e., the evaluation of 
metamodels or the analysis of conceptual models.

This research guides the improvement of our teaching material - not only for the Smart City case. We aim to revise the material and extend it in a way that enables its provision as a massive open online course (MOOC). Online courses come with distinct additional requirements and challengers [48], also particularly for conceptual modeling (cf. [49]). We plan to integrate all learning material including lecture slides, practical exercises, video tutorials among others within the Open Models Laboratory (OMiLAB, www.omilab.org). This will eventually enable the worldwide OMiLAB community [19] to access, use, and adopt the Smart City case. Moreover, the OMiLAB learning environment could be enriched with immediate and personalized feedback as proposed by Serral et al. [2].

\section{References}

[1] D. Bogdanova and M. Snoeck, "Domain Modelling in Bloom: Deciphering How We Teach It," in IFIP Working Conference on The Practice of Enterprise Modeling, pp. 3-17, Springer, 2017.

[2] E. Serral, J. De Weerdt, G. Sedrakyan, and M. Snoeck, "Automating Immediate and Personalized Feedback Taking Conceptual Modelling Education to a Next Level," in Research Challenges in Information Science (RCIS), 2016 IEEE Tenth International Conference on, pp. 1-6, IEEE, 2016.

[3] S. Strecker, D. Kundisch, F. Lehner, J. M. Leimeister, and P. Schubert, "Higher Education and the Opportunities and Challenges of Educational Technology," Business \& Information Systems Engineering, vol. 60, no. 2, pp. 181-189, 2018.

[4] B. Ternes and S. Strecker, "A web-based modeling tool for studying the learning of conceptual modeling," Modellierung 2018, 2018.

[5] U. Frank, S. Strecker, P. Fettke, J. vom Brocke, J. Becker, and E. Sinz, "The research field modeling business information systems," Business \& Information Systems Engineering, vol. 6, no. 1, pp. 39-43, 2014.

[6] R. Jung and C. Lehrer, "Guidelines for Education in Business and Information Systems Engineering at Tertiary Institutions," Business \& Information Systems Engineering, vol. 59, no. 3, pp. 189-203, 2017.

[7] J. Mylopoulos, "Conceptual modelling and telos," Conceptual modeling, databases, and CASE, pp. 363-376, 2008.

[8] M. Daun, A. Salmon, B. Tenbergen, T. Weyer, and K. Pohl, "Industrial case studies in graduate requirements engineering courses: The impact on student motivation," in Software Engineering Education and Training (CSEE\&T), 2014 IEEE 27th Conference on, pp. 3-12, IEEE, 2014.

[9] D. Karagiannis and H. Kühn, "Metamodeling Platforms," in Third International Conference EC-Web 2002 Dexa 2002 (K. Bauknecht, A. Min Tjoa, and G. Quirchmayr, eds.), (Aix-en-Provence, France), p. 182, Springer, 2002.
[10] D. R. Krathwohl, "A revision of Bloom's taxonomy: An overview," Theory into practice, vol. 41, no. 4, pp. 212-218, 2002.

[11] B. S. Bloom, M. D. Engelhart, E. J. Furst, W. H. Hill, D. R. Krathwohl, et al., "Taxonomy of educational objetives: the classification of educational goals: handbook I: cognitive domain," tech. rep., New York, US: D. Mckay, 1956.

[12] M. Naphade, G. Banavar, C. Harrison, J. Paraszczak, and R. Morris, "Smarter Cities and Their Innovation Challenges," Computer, vol. 44, no. 6, pp. 32-39, 2011.

[13] R. Barnett, "Knowing and becoming in the higher education curriculum," Studies in higher education, vol. 34, no. 4, pp. 429-440, 2009.

[14] D. Bork and H.-G. Fill, "Formal Aspects of Enterprise Modeling Methods: A Comparison Framework," in System Sciences (HICSS), 2014 47th Hawaii International Conference on, pp. 3400-3409, IEEE, 2014.

[15] U. Frank, "Domain-Specific Modeling Languages: Requirements Analysis and Design Guidelines," in Domain Engineering, pp. 133-157, Springer, 2013.

[16] D. Karagiannis, H. C. Mayr, and J. Mylopoulos, Domain-Specific Conceptual Modelling. Springer, 2016.

[17] D. Götzinger, E. Miron, and F. Staffel, "OMiLAB: An Open Collaborative Environment for Modeling Method Engineering," in Domain-Specific Conceptual Modeling, Concepts, Methods and Tools, pp. 55-76, 2016.

[18] D. Karagiannis, "Agile modeling method engineering," in Proceedings of the 19th Panhellenic Conference on Informatics, pp. 5-10, ACM, 2015.

[19] D. Bork and E.-T. Miron, "OMiLAB - An Open Innovation Community for Modeling Method Engineering," in 8th International Conference of Management and Industrial Engineering (ICMIE'2017) (A. Niculescu, O. D. Negoita, and B. Tiganoaia, eds.), pp. 64-77, 2017.

[20] N. Efendioglu, R. Woitsch, and W. Utz, "A Toolbox Supporting Agile Modelling Method Engineering: ADOxx. org Modelling Method Conceptualization Environment," in IFIP Working Conference on The Practice of Enterprise Modeling, pp. 317-325, Springer, 2016.

[21] D. Bork, H.-G. Fill, D. Karagiannis, E.-T. Miron, N. Tantouris, and M. Walch, "Conceptual Modelling for Smart Cities: A Teaching Case," Interaction Design and Architectures (IxD\&A), vol. 27, pp. 10-28, 2015.

[22] D. Bork, R. Buchmann, I. Hawryszkiewycz, D. Karagiannis, N. Tantouris, and M. Walch, "Using Conceptual Modeling to Support Innovation Challenges in Smart Cities," in 14th IEEE International Conference on Smart City, pp. 1317-1324, 2016.

[23] N. B. Zaidi, C. Hwang, S. Scott, S. Stallard, J. Purkiss, and M. Hortsch, "Climbing Bloom's taxonomy pyramid: Lessons from a graduate histology course," Anatomical sciences education, vol. 10, no. 5, pp. 456-464, 2017.

[24] D. Karagiannis, R. A. Buchmann, and D. Bork, "MANAGING CONSISTENCY IN MULTI-VIEW ENTERPRISE MODELS: AN APPROACH BASED ON SEMANTIC QUERIES," in Proceedings of the Twenty-Fourth European Conference on Information Systems (ECIS), Istanbul, Turkey, 2016. Research Papers. 53. 
[25] A. Awadid, D. Bork, D. Karagiannis, and S. Nurcan, "Toward generic consistency patterns in multi-view enterprise modelling," in Twenty-Sixth European Conference on Information Systems (ECIS'2018), 2018.

[26] J. Krogstie, O. I. Lindland, and G. Sindre, "Defining quality aspects for conceptual models," in Information System Concepts, pp. 216-231, Springer, 1995.

[27] S. Strahringer, "Ein sprachbasierter Metamodellbegriff und seine Verallgemeinerung durch das Konzept des Metaisierungsprinzips," in Modellierung, vol. 98, pp. 15-20, 1998.

[28] H. Kern, A. Hummel, and S. Kühne, "Towards a comparative analysis of meta-metamodels," in Proceedings of the compilation of the co-located workshops on DSM'11, TMC'11, AGERE! 2011, AOOPES'11, NEAT'11, \& VMIL'11, pp. 7-12, ACM, 2011.

[29] D. Bork, "Metamodel-based Analysis of Domain-specific Conceptual Modeling Methods,' in 11th IFIP WG 8.1 working conference on the Practice of Enterprise Modelling (PoEM) (R. Buchmann, D. Karagiannis, and M. Kirikova, eds.), p. in press, 2018.

[30] J. Bézivin, F. Jouault, P. Rosenthal, and P. Valduriez, "Modeling in the large and modeling in the small," in Model Driven Architecture, pp. 33-46, Springer, 2005.

[31] P. Höfferer, "Achieving Business Process Model Interoperability Using Metamodels and Ontologies," in ECIS, pp. 1620-1631, 2007.

[32] R. A. Buchmann and D. Karagiannis, "Enriching Linked Data with Semantics from Domain-Specific Diagrammatic Models," Business \& Information Systems Engineering, vol. 58, no. 5, pp. 341-353, 2016.

[33] D. Karagiannis, R. A. Buchmann, and D. Bork, "Managing Consistency in Multi-View Enterprise Models: an Approach based on Semantic Queries," in 24th European Conference on Information Systems, ECIS 2016, Istanbul, Turkey, June 12-15, 2016 , p. Research Paper 53, 2016.

[34] J. Di Rocco, D. Di Ruscio, L. Iovino, and A. Pierantonio, "Mining Metrics for Understanding Metamodel Characteristics," in Proceedings of the 6th International Workshop on Modeling in Software Engineering, MiSE 2014, (New York, NY, USA), pp. 55-60, ACM, 2014.

[35] G. Hinkel, M. Kramer, E. Burger, M. Strittmatter, and L. Happe, "An empirical study on the perception of metamodel quality," in Model-Driven Engineering and Software Development (MODELSWARD), 2016 4th International Conference on, pp. 145-152, IEEE, 2016.

[36] D. Moody, "The 'physics' of notations: toward a scientific basis for constructing visual notations in software engineering," IEEE Transactions on Software Engineering, vol. 35, no. 6, pp. 756-779, 2009.

[37] D. Bork, D. Karagiannis, and B. Pittl, "How are Metamodels Specified in Practice? Empirical Insights and Recommendations," in Twenty-fourth Americas Conference on Information Systems, pp. 1-10, 2018.

[38] H.-G. Fill and D. Karagiannis, "On the conceptualisation of modelling methods using the ADOxx meta modelling platform," Enterprise Modelling and Information Systems Architectures-An International Journal, vol. 8, no. 1, 2013.
[39] Á. Lédeczi, G. Nordstrom, G. Karsai, P. Volgyesi, and M. Maroti, "On metamodel composition," in Control Applications, 2001.(CCA'01). Proceedings of the 2001 IEEE International Conference on, pp. 756-760, IEEE, 2001.

[40] D. Karagiannis, "Conceptual Modelling Methods: the AMME Agile Engineering Approach," in Proceedings of the 9th International Management Conference, Springer, 2017.

[41] D. Bork, "Using Conceptual Modeling for Designing Multi-View Modeling Tools," in 21st Americas Conference on Information Systems, AMCIS 2015, Puerto Rico, 2015.

[42] H. Chourabi, T. Nam, S. Walker, J. R. Gil-García, S. Mellouli, K. Nahon, T. A. Pardo, and H. J. Scholl, "Understanding Smart Cities: An Integrative Framework," in 45th Hawaii International International Conference on Systems Science (HICSS-45), Proceedings, Maui, HI, USA, pp. 2289-2297, IEEE Computer Society, 2012.

[43] M. Kehoe, M. Cosgrove, S. Gennaro, C. Harrison, W. Harthoorn, J. Hogan, J. Meegan, P. Nesbitt, and C. Peters, "Smarter Cities Series: A Foundation for Understanding IBM Smarter Cities," Redguides for Business Leaders, IBM, 2011.

[44] M. Batty, K. W. Axhausen, F. Giannotti, A. Pozdnoukhov, A. Bazzani, M. Wachowicz, G. Ouzounis, and Y. Portugali, "Smart cities of the future," The European Physical Journal Special Topics, vol. 214, no. 1, pp. 481-518, 2012.

[45] S. Alawadhi, A. Aldama-Nalda, H. Chourabi, J. R. Gil-Garcia, S. Leung, S. Mellouli, T. Nam, T. A. Pardo, H. J. Scholl, and S. Walker, "Building understanding of smart city initiatives," in International Conference on Electronic Government Electronic Government: 11th IFIP WG 8.5 International Conference, EGOV 2012, Kristiansand, Norway, Proceedings (H. J. Scholl, ed.), pp. 40-53, Springer Berlin Heidelberg, 2012.

[46] D. Topetta, "The Smart City Vision: How Innovation and ICT can build smart, "liveable", sustainable cities," 2010.

[47] F. J. Riggins and S. F. Wamba, "Research Directions on the Adoption, Usage, and Impact of the Internet of Things through the Use of Big Data Analytics," in 48th Hawaii International Conference on System Sciences, HICSS 2015, Proceedings, Kauai, HI, USA (T. X. Bui and R. H. S. Jr., eds.), pp. 1531-1540, IEEE Computer Society, 2015.

[48] R. Kop, "The challenges to connectivist learning on open online networks: Learning experiences during a massive open online course," The International Review of Research in Open and Distributed Learning, vol. 12, no. 3, pp. 19-38, 2011.

[49] M. Daun, J. Brings, P. A. Obe, K. Pohl, S. Moser, H. Schumacher, and M. Rieß, "Teaching Conceptual Modeling in Online Courses: Coping with the Need for Individual Feedback to Modeling Exercises," in Software Engineering Education and Training (CSEE\&T), 2017 IEEE 30th Conference on, pp. 134-143, IEEE, 2017. 